\title{
Borda Count in Collective Decision Making: A Summary of Recent Results
}

\author{
Jörg Rothe \\ Institut für Informatik \\ Heinrich-Heine-Universität Düsseldorf \\ 40225 Düsseldorf, Germany \\ rothe@cs.uni-duesseldorf.de
}

\begin{abstract}
Borda Count is one of the earliest and most important voting rules. Going far beyond voting, we summarize recent advances related to Borda in computational social choice and, more generally, in collective decision making. We first present a variety of well known attacks modeling strategic behavior in voting -including manipulation, control, and bribery - and discuss how resistant Borda is to them in terms of computational complexity. We then describe how Borda can be used to maximize social welfare when indivisible goods are to be allocated to agents with ordinal preferences. Finally, we illustrate the use of Borda in forming coalitions of players in a certain type of hedonic game. All these approaches are central to applications in artificial intelligence.
\end{abstract}

\section{Introduction}

More than 230 years ago, Borda (1781) proposed one of the most important and influential voting rules up to date. It is simple and strikingly elegant: When there are $m$ candidates, the voters rank them by a linear order according to their preferences; a candidate in $i$ th position of a voter's ranking scores $m-i$ points; and the candidates with the most points win. Borda and its modifications have been widely used in political elections (e.g., in Slovenia or to elect the leader of the Irish Green Party) or by academic institutions. For instance, the French Academy of Sciences adopted this rule to elect its members for about two decades in the 18th century. The debates between the Chevalier de Borda and the Marquis de Condorcet, both members of this Academy, about whose voting method is better are legendary. Social choice theorists have continued to fiercely dispute this question up to now; for example, Saari (2003; 2006) champions Borda, a view that Risse (2005) strongly disagrees with. The goal of this survey, however, is not a social-choice-theoretic treatise of Borda compared with other voting rules; ${ }^{1}$ rather, our goal is to summarize recent advances related to Borda within the field of computational social choice and, going far beyond voting alone, collective

Copyright (c) 2019, Association for the Advancement of Artificial Intelligence (www.aaai.org). All rights reserved.

${ }^{1}$ For more details about the social-choice-theoretic properties of Borda, we refer to the recent book chapters by Zwicker (2016) and Baumeister and Rothe (2015). decision making in general, such as the allocation of indivisible goods and coalition formation in hedonic games.

The first and foremost purpose of this paper is to present the standard attacks that have been proposed within computational social choice to model strategic behavior in voting, such as manipulation, control, and bribery attacks of various types. While we will focus on Borda's voting rule to illustrate each such attack and to discuss how resistant Borda is to them in terms of computational complexity, we will also mention in passing some related results for other voting rules. This will give an overview of some of the most exciting work in computational social choice-having evolved to become an established subarea of distributed AI and multiagent systems-during the last decades (see Section 2).

Next, in Section 3, we leave voting behind and first show how Borda can be used to maximize social welfare when indivisible goods are to be allocated to agents with ordinal preferences. Then we turn to hedonic games where a number of players, each having preferences over the coalitions they can join, are faced with the problem of coalition formation. Again, we will show how Borda can be used in this context and present some recent results. All three fieldspreference aggregation by voting, the allocation of indivisible goods, and hedonic games - are central to certain AI applications. Voting, for example, has been employed in AI subareas as diverse as automated scheduling (Haynes et al. 1997), collaborative filtering (Pennock, Horvitz, and Giles 2000), computational linguistics (Oflazer and Tür 1997), information extraction (Sigletos et al. 2005), planning (Ephrati and Rosenschein 1997), recommender systems (Ghosh et al. 1999), and web searching (Dwork et al. 2001).

Finally, we highlight some open questions in Section 4.

\section{How Resistant is Borda to Manipulative Attacks in Voting?}

In this section we survey some of the most exciting work in computational social choice of the previous decades: the study of strategic behavior in voting, and how computational complexity can be used as a barrier against such attacks that aim at influencing the outcome of an election. Three basic attack types are distinguished in the literature: manipulation, control, and bribery. While focusing on Borda when explaining them, we will also consider various other voting rules. 


\subsection{Manipulation}

Suppose we are given an election $(C, V)$ with five candidates, $C=\{a, b, c, d, e\}$, and the following list of five votes in $V$, each cast by an honest voter:

$$
\begin{array}{llllllllllll}
v_{1}: & d & c & a & e & b & v_{2}: & d & c & b & a & e \\
v_{3}: & d & b & e & a & c & v_{4}: & e & c & b & a & d \\
v_{5}: & c & b & d & a & e & & & & & &
\end{array}
$$

where a vote like $v_{1}$ 's ( $d c a$ e $b$ ) means that $v_{1}$ prefers $d$ to $c, c$ to $a$, etc. Using Borda in $(C, V), a$ scores 6 points, $b 10$ points, $c 13$ points, $d 14$ points, and $e 7$ points, so $d$ alone wins. Now suppose that an insincere voter, $v_{6}$ whose truthful vote is $c d b a e$, joins the election. Knowing the other voters' preferences, however, $v_{6}$ strategically casts the vote $c$ a e $b d$, so $c$ alone wins the election $\left(C, V \cup\left\{v_{6}\right\}\right)$ with a score of 17 , while $a, b, d$, and $e$ now have $9,11,14$, and 9 points, respectively. (Casting $v_{6}$ 's truthful vote would have made both $c$ and $d$ win the election with 17 points, but $v_{6}$ wants to make sure that her favorite candidate $c$ is the only winner.) Thus $v_{6}$ has successfully manipulated the election.

Motivated by a famous result of Gibbard (1973) and Satterthwaite (1975) (which, roughly speaking, says that every reasonable voting rule is manipulable), Bartholdi III, Tovey, and Trick (1989) proposed to use computational complexity to prevent manipulation from happening or being successful. They defined the constructive manipulation problem (CM): Given an election $(C, V)$, a distinguished candidate $c \in C$, and a strategic voter $s$, is it possible for $s$ to cast a vote such that $c$ is the winner of the election $(C, V \cup\{s\})$ ? For Borda, though, they showed that this problem is easy to solve: A simple greedy algorithm solves CM for Borda in polynomial time. In fact, this greedy algorithm works for every voting rule that can be represented by a scoring function that is both responsive (i.e., candidates with highest score win) and monotonic (i.e., moving a candidate to a better position in a preference ranking cannot result in this candidate scoring fewer points). On the other hand, they showed that another voting rule, "second-order Copeland," resists manipulation in the sense that CM for it is NP-complete. Bartholdi III and Orlin (1991) established that for the voting rule STV ("single transferrable vote") CM is NP-complete as well. ${ }^{2}$

Conitzer, Sandholm, and Lang (2007) generalize CM in two ways: First, they allow voters to be weighted and, second, they consider coalitions of manipulators, leading to the constructive coalitional weighted manipulation problem (CCWM), where the weights and preferences of the honest voters but only the manipulators' weights are given. For example, suppose that in the election given above $v_{1}, \ldots, v_{4}$ are honest voters with weight 2 each, whereas $v_{5}$ and $v_{6}$ both have weight 1 and form a coalition of manipulators who wish to make their favorite candidate, $c$, win. Casting their truthful votes, $c b d a e$ for $v_{5}$ and $c d b a e$ for $v_{6}$, would result in $d$ (with 29 points) beating $c$ (with 26 points) and also $a, b$, and $e$ (with even fewer points). But if $v_{5}$ and $v_{6}$ cast strategic votes (e.g., both $c a b$ e d), they would make $c$ (still

\footnotetext{
${ }^{2}$ Rothe and Schend (2013) note that the reduction of Bartholdi III and Orlin (1991) is slightly flawed but can be easily fixed.
}

with a score of 26 points, but $d$ now scoring only 24 points and $a, b$, and $e$ even less) the Borda winner of the election.

Conitzer, Sandholm, and Lang (2007) show that CCWM is NP-complete for Borda even when there are only three candidates (and is in $\mathrm{P}$ for up to two candidates). Similar results for CCWM have been obtained for many other voting rules, such as plurality, veto, Copeland, maximin, and STV (Conitzer, Sandholm, and Lang 2007), Baldwin's and Nanson's variants of Borda that we will again consider later on (Davies et al. 2014), and Bucklin and fallback voting (Faliszewski et al. 2015) (we omit defining all these rules due to space limitations, but refer to the book chapters by Conitzer and Walsh (2016) and Baumeister and Rothe (2015)). An interesting special case occurs for the Copeland rule ${ }^{3}$ with three candidates: While CCWM for it is in $\mathrm{P}$ for the uniquewinner model (which requires the distinguished candidate $c$ to be the only winner for the manipulation attack to be successful), Faliszewski, Hemaspaandra, and Schnoor (2008) show that this problem is NP-complete in the nonuniquewinner model (where $c$ may be one among several winners for the manipulation attack to be successful). The known complexity results for CCWM with respect to all other voting rules considered are the same in both winner models.

Borda is a very prominent member of a whole class of important voting rules, the so-called scoring protocols, that also contains plurality and veto. A scoring protocol for $m$ candidates is defined by a scoring vector $\sigma=\left(\sigma_{1}, \ldots, \sigma_{m}\right)$ of nonnegative integers, $\sigma_{1} \geq \cdots \geq \sigma_{m}$, where a candidate in the $i$ th position of a vote gets $\sigma_{i}$ points, and whoever has the most points wins. Borda is thus defined via $(m-1, m-2, \ldots, 0)$, plurality via $(1,0, \ldots, 0)$, and veto via $(1, \ldots, 1,0)$. Hemaspaandra and Hemaspaandra (2007) established the following dichotomy result (which for the case of three candidates was also observed by Conitzer, Sandholm, and Lang (2007)): CCWM is in P for plurality and the trivial scoring protocol with vector $(0, \ldots, 0)$, and is NPcomplete for all other scoring protocols.

So far we have considered only the constructive case where the goal of the manipulator(s) is to make a given candidate win. Conitzer, Sandholm, and Lang (2007) were the first to define the destructive variant where the goal is to block a given candidate's victory. The destructive analogue of CCWM, denoted by DCWM, has also been studied for most of the voting rules mentioned above. It turns out that DCWM is never harder than CCWM, but it can be easier. For example, Conitzer, Sandholm, and Lang (2007) showed that DCWM for Borda is in P, and this holds true for each voting rule that can be represented by a scoring function that is both responsive and monotonic and whose winners can be determined in polynomial time. By contrast, they also showed that DCWM for STV (which is not monotonic) is NP-complete even for three candidates.

Coming back to constructive manipulation for Borda, we have seen that CCWM is NP-hard, yet CM is easy to solve.

\footnotetext{
${ }^{3}$ In a Copeland election, each candidate who is preferred to another candidate by a majority of voters earns one point in this headon-head contest; for each tie, they both earn half a point; and the candidates with the most points win.
} 
But what about the intermediate case, the case where voters are unweighted and still there is a coalition of manipulators? Denote this problem by CCUM. Its complexity for Borda has been a mystery for several years. Then, in 2011, two papers resolved this open question independently at about the same time: Betzler, Niedermeier, and Woeginger (2011) and Davies et al. $(2011 ; 2014)$ showed that CCUM is NPcomplete even when there are only two manipulators. Indeed, this was one of the greatest moments in computational social choice: Betzler, Niedermeier, and Woeginger (2011) presented their work in the IJCAI 2011 Distinguished Papers session, and Davies et al. (2011) were honored by an AAAI 2011 Outstanding Paper Award.

Zuckerman, Procaccia, and Rosenschein (2009) considered an optimization variant of CCUM, denoted by CCUO: Given the unweighted votes of sincere voters and a distinguished candidate $c$, determine the minimum number of manipulators needed in order to make $c$ win. They designed an efficient algorithm that approximates CCUO for Borda up to an additive error of one. They also studied the weighted variant, noting that a shortcoming of NP-hardness results is that they are worst-case complexity results only, thus providing a "poor obstacle against potential manipulators," as these may still be able to succeed in typical settings. Instead, Zuckerman, Procaccia, and Rosenschein (2009) took a different approach: They designed efficient heuristics, characterized "small windows" of instances where these may fail, and proved that they are correct on all other instances. For Borda, they showed that if there is a manipulation for an instance with certain weights, their heuristics will succeed when given an extra manipulator with maximal weight. Rothe and Schend (2013) survey this and other approaches to dealing with challenges to complexity shields that are supposed to protect elections against manipulative attacks.

\subsection{Control}

While one may feel a bit uneasy about manipulators strategically changing the outcome of an election, there is actually not much one could put forward against it. After all, every voter-human or software agent-has the right to think strategically about which vote to cast; not doing so would not be smart. Electoral control, however, is better suitable than manipulation as a model of electoral fraud or vote rigging - in the sense of acts that are considered ethically unacceptable, outside the spirit of an election, or in violation of the principles of democracy. Here we assume that an external authority, called the (election) chair, seeks to influence the outcome of an election via exerting certain control actions. Bartholdi III, Tovey, and Trick (1992) were the first to introduce control attacks (such as constructive control by deleting voters) and their associated decision problems (CCDV): Given an election $(C, V)$, a distinguished candidate $c \in C$, and a nonnegative integer $k$, is it possible for the chair to make $c$ win by deleting up to $k$ votes from $V$ ? For example, in the election $(C, V)$ considered above, with $C=\{a, b, c, d, e\}$ and $V=\left(v_{1}, \ldots, v_{5}\right)$, we have seen that $d$ is the only Borda winner. However, by deleting just one voter, namely $v_{3}$, the chair can ensure that now $c$ alone wins.

The other control actions/problems studied by Bartholdi
III, Tovey, and Trick (1992) (which due to space limitations will not be defined in detail here) are constructive control by adding voters (CCAV), constructive control by partition of voters (CCPV), constructive control by deleting candidates (CCDC), constructive control by adding candidates (CCAC), constructive control by partition of candidates (CCPC), and constructive control by run-off partition of candidates (CCRPC). Each such control type captures a particular way of rigging elections. For example, CCDV models voter disenfranchisement; by adding spoiler candidates in CCAC, the chair seeks to weaken the rivals of her favorite candidate; and partition of voters in CCPV (which is formalized as a two-stage election where the electorate is partitioned to create two subelections whose winners face each other in the final run-off) is a (rather simple) model of gerrymandering, a common practice to achieve an advantage from suitably shifting the boundaries of voting districts. ${ }^{4}$ Similar scenarios motivate the other control types.

The control-by-partition cases come in two variants each by using a rule that specifies how to handle ties in their firststage subelections: Either all subelection winners move forward to the final run-off (ties promote, TP) or only unique subelection winners move forward (ties eliminate, TE). This distinction is due to Hemaspaandra, Hemaspaandra, and Rothe (2007), who also introduce the destructive analogues of these control types: DCDV, DCAV, DCPV, DCDC, DCAC, DCPC, and DCRPC. ${ }^{5}$

Bartholdi III, Tovey, and Trick (1992) classified the complexity of the constructive control problems for Condorcet voting and plurality; Hemaspaandra, Hemaspaandra, and Rothe (2007) did so for their destructive variants, and also for constructive and destructive control in approval voting; Faliszewski et al. (2009) studied the complexity of control for Copeland ${ }^{\alpha}, 6$ Menton (2013) for (normalized) range voting; and Erdélyi et al. (2015) for Bucklin and fallback voting. Interestingly, unlike for manipulation, some voting rules are immune to certain control actions. For example, Condorcet is immune to constructive control by adding candidates and to destructive control by deleting or partitioning candidates, and the same applies to plurality and range voting. If a voting rule it not immune to a control type, it is susceptible to it, and in this case it makes sense to determine the complexity of the corresponding control problem. Among natural voting rules with a winner problem in $\mathrm{P}$,

\footnotetext{
${ }^{4}$ More natural or sophisticated models of gerrymandering were considered, e.g., by Puppe and Tasnádi (2009), Erdélyi, Hemaspaandra, and Hemaspaandra (2015), and Bachrach et al. (2016).

${ }^{5}$ Hemaspaandra, Hemaspaandra, and Menton (2013) observed that, depending on the tie-handling rule (TP vs. TE) and the winner model (nonunique vs. unique winner) used, DCPC and DCRPC can be the same problem. The difference between control by partition and by run-off partition of candidates is that in the latter the winners of both subelections run against each other in the final runoff, whereas in the former the winners of one subelection face all candidates of the other subelection in the final round.

${ }^{6}$ Copeland $^{\alpha}$ generalizes Copeland by rewarding each tie between candidates in a head-on-head contest with $\alpha$ points, $\alpha \in$ $\mathbb{Q} \cap[0,1]$. Copeland $^{0.5}$ is the common Copeland rule (see Footnote 3 ). Copeland ${ }^{1}$ was proposed by Ramon Llull as early as 1299 .
} 
Table 1: Overview of control complexity in Borda elections

\begin{tabular}{lcl}
\hline Control type & Result & Source \\
\hline CCDV & NPC & Hemaspaandra and Schnoor (2016) \\
CCAV & NPC & Russel (2007) \\
CCPV-TP & NPC & Neveling and Rothe (2017a) \\
CCPV-TE & NPC & Neveling and Rothe (2017b) \\
\hline CCDC & NPC & Chen et al. (2015) \\
CCAC & NPC & Elkind, Faliszewski, and Slinko (2011) \\
CCPC-TP & NPC & Neveling and Rothe (2017a) \\
CCPC-TE & NPC & Neveling and Rothe (2017a) \\
CCRPC-TP & NPC & Neveling and Rothe (2017a) \\
CCRPC-TE & NPC & Neveling and Rothe (2017b) \\
\hline DCDV & P & Russel (2007) \\
DCAV & P & Russel (2007) \\
DCPV-TP & NPC & Neveling and Rothe (2017a) \\
DCPV-TE & P & Russel (2007) \\
\hline DCDC & P & Loreggia et al. (2015) \\
DCAC & P & Loreggia et al. (2015) \\
DCPC-TP & $?$ & \\
DCPC-TE & P & Neveling and Rothe (2017a) \\
DCRPC-TP & NPC & Neveling and Rothe (2017a) \\
DCRPC-TE & P & Neveling and Rothe (2017a) \\
\hline
\end{tabular}

normalized range voting (Menton 2013) and fallback voting (Erdélyi et al. 2015) display the broadest resistance (in the sense of NP-completeness, denoted by NPC) to control currently known: They are vulnerable (i.e., the associated control problem is in P) to only two control types (DCDV and DCAV) and resistant in all other cases. Due to space limitations, we omit stating all related results explicitly (or citing all papers with results on the complexity of control) but instead refer to the book chapters by Faliszewski and Rothe (2016) and Baumeister and Rothe (2015).

What about the control complexity in Borda elections? A closer look at the two book chapters just mentioned reveals that only nine of the many control scenarios had been solved for Borda by 2016. However, almost all other cases could be settled in two recent papers by Neveling and Rothe (2017b; 2017a). Table 1 gives an overview of the control complexity in Borda elections in the unique-winner model. ${ }^{7}$ Borda is resistant to all constructive control cases, and is vulnerable in all destructive control cases except DCRPC-TP and DCPV-TP. The complexity of DCPC-TP, indicated by a question mark, is still open. Interestingly, Neveling and Rothe (2017a) show that Borda is vulnerable to this control type (i.e., DCPC-TP is in P) in the nonunique-winner model. As a consequence of Footnote 5, DCRPC-TP for Borda is in $\mathrm{P}$ in the nonunique-winner model as well, yet is NP-hard in the unique-winner model. Again, Rothe and Schend (2013) survey various approaches to dealing with challenges to NP-hardness shields against control attacks.

Neveling and Rothe (2017b) also consider online can-

\footnotetext{
${ }^{7} \mathrm{NP}$-completeness of CCDV has been shown by Neveling and Rothe (2017b) as well; however, as they learned later, this already follows from a dichotomy result of Hemaspaandra and Schnoor (2016) for this problem in the class of scoring protocols.
}

didate control in sequential Borda elections-a dynamic, partial-information model due to Hemaspaandra, Hemaspaandra, and Rothe (2017) where the candidates show up in sequence, one after the other, the votes being gradually extended to add the current candidate in each step, and the chair must decide right now whether or not to exert the given control action (e.g., to either delete the current candidate now or never). That is, the chair has a "use-it-or-lose-it ability" to exert control. Extending the corresponding results for sequential plurality due to Hemaspaandra, Hemaspaandra, and Rothe (2017), Neveling and Rothe (2017b) show that sequential Borda is vulnerable to online constructive and destructive control by either adding or deleting candidates.

\subsection{Bribery}

Another way to fiddle around with elections so as to change their outcome to one's own advantage is bribery, a model proposed by Faliszewski, Hemaspaandra, and Hemaspaandra (2009), see also (Faliszewski et al. 2009): A briber seeks to influence the outcome of an election by bribing certain voters without exceeding a given budget. Bribery shares certain features with manipulation and others with control, e.g., the briber is an external actor who needs to choose which votes to affect as in control, and as in manipulation the briber needs to find suitable preference orders when changing these bribed votes. In the decision problem associated with the most basic variant of bribery, denoted by BRIBERY, we are given an election $(C, V)$, a distinguished candidate $c \in C$, a budget $B \in \mathbb{N}$, and a collection $\left(\chi_{1}, \ldots, \chi_{n}\right)$ of cost functions, one for each voter. For each $i, 1 \leq i \leq n$, and each preference order over $C, \chi_{i}$ gives the cost of convincing the $i$ th voter to cast this preference order instead of her original one, where we assume that keeping the original vote always has zero cost. We ask whether there is a preference profile $V^{\prime}$ such that $c$ wins in $\left(C, V^{\prime}\right)$ and the sum of the costs of changed votes doesn't exceed $B$. While "bribery" commonly has a rather negative connotation, it can also be positively interpreted, as, e.g., Faliszewski et al. (2015) do, in terms of "campaign management" where the manager of a political campaign seeks to convince voters to change their votes and these efforts have certain costs.

For an example, look again at the election $(C, V)$ considered above, with $C=\{a, b, c, d, e\}, V=\left(v_{1}, \ldots, v_{5}\right)$, and the Borda winner $d$ scoring 14 points. Assume that $a$ (with 6 points currently) is our distinguished candidate, the budget is 2 , and all voters have unit cost. Then $a$ can be made a unique Borda winner by bribing, e.g., $v_{2}$ and $v_{3}$ to change their votes to $a e b c d$ and $a e b d c$, yielding a score of 12 for $a$, of 9 for $b$, of 11 for $c$, of 7 for $d$, and of 11 for $e$. If the budget were 1 , though, no bribery action would be successful, as by bribing only one voter, $a$ could gain only 3 points (giving a score of at most 9), but $d$ could lose no more than four points (giving a score of at least 10).

Among many other results, Faliszewski, Hemaspaandra, and Hemaspaandra (2009) established a dichotomy result in the class of scoring protocols: For each $\sigma=\left(\sigma_{1}, \ldots, \sigma_{m}\right)$, if $\sigma_{2}=\cdots=\sigma_{m}$ then the weighted variant of BRIBERY for $\sigma$ is in P; otherwise, it is NP-complete. In particular, weighted BRIBERY for Borda with three or more candidates 
is NP-complete. Brelsford et al. (2008) proved that even in the unweighted case, BRIBERY for Borda is NP-complete and also provided an inapproximability result for bribery.

Elkind, Faliszewski, and Slinko (2009) defined another variant of the bribery problem, denoted SWAP-BRIBERY (which generalizes the manipulation problem CCUM considered earlier), where the briber has to pay for each individual swap of adjacent candidates in the votes separately. They showed that SWAP-BRIBERY for Borda (and many other voting rules) is NP-complete. That was why they also introduced the special variant SHIFT-BRIBERY, which is defined like SWAP-BRIBERY except that each swap must involve the distinguished candidate. Still, they showed that SHIFTBRIBERY for Borda is NP-complete, yet can be efficiently approximated to within a factor of 2 (which was generalized by Elkind and Faliszewski (2010) for all scoring protocols). Recently, Maushagen et al. (2018) studied the complexity of SHIFT-BRIBERY for iterative voting rules such as those by Baldwin (1926) and Nanson (1882), and showed that they are NP-complete as well. These two voting rules proceed in rounds and eliminate in each round the candidates performing worst (namely, the candidates with lowest Borda score in Baldwin and those with scores lower than the average Borda score in Nanson), and the remaining candidates win.

The complexity of bribery has been studied for many other voting rules as well; for instance, Faliszewski et al. (2009) studied bribery in Copeland ${ }^{\alpha}$ elections. Due to space limitations, we again omit stating all these results and papers, referring to the book chapters by Faliszewski and Rothe (2016) and Baumeister and Rothe (2015) instead.

\section{Using Borda beyond Voting}

We now leave voting behind and turn to two recent applications of the Borda rule in other fields of collective decision making: social welfare maximization in the allocation of indivisible goods and coalition formation in hedonic games.

\subsection{Allocation of Indivisible Goods}

Allocating indivisible goods to agents having preferences over (bundles of) goods is an important field at the intersection of $\mathrm{AI}$ and economics. There are tons of literature on the allocation of indivisible goods, which cannot all be cited here; instead we refer to the book chapters by Bouveret, Chevaleyre, and Maudet (2016) and Lang and Rothe (2015).

Let $N=\{1, \ldots, n\}$ be a set of agents and $G$ a set of $m$ goods. An allocation of $G$ to $N$ is a partition $\left(\pi_{1}, \ldots, \pi_{n}\right)$ of $G$ (i.e., $G=\bigcup_{i=1}^{n} \pi_{i}$ and $\pi_{i} \cap \pi_{j}=\emptyset$ for $i \neq j$ ), where $\pi_{i}$ is the bundle of goods assigned to agent $i$. A common approach to how agents value their bundles is to assume additive preferences: Every agent $i$ assigns a positive number to each good and $i$ 's utility for a bundle of goods is the sum of the corresponding numbers. Here, however, we take a different approach: We assume that agents have ordinal preferences over $G$, i.e., a ranking of the goods, and the agents' utilities are now specified by a fixed, agent-independent vector that maps ranks into scores just as in voting. In particular, Brams, Edelman, and Fishburn (2003) (and later Brams and King (2005) and Bouveret, Endriss, and Lang (2010)) studied Borda-optimal allocations. ${ }^{8}$ Baumeister et al. (2017) generalized these by introducing scoring allocation correspondences, which informally stated proceed in three steps: First, we use a scoring vector $\sigma=\left(\sigma_{1}, \ldots, \sigma_{m}\right)$ to derive from the agents' preferences a utility vector for each possible allocation $\pi$, thus specifying each agent's individual utility for $\pi$. Second, these individual utilities are aggregated via an aggregation function (typically, via utilitarian or egalitarian social welfare, i.e., by using the sum or the minimum of the agents' individual utilities) to obtain the collective utility of $\pi$. Third, we choose the outcomes $\pi$ that maximize collective utility. (If desired, one can break ties so as to yield a scoring allocation rule, which always outputs only one allocation $\pi$ maximizing collective utility.)

Brams, Edelman, and Fishburn (2003) study properties of Borda-optimal allocations such as envy-freeness (i.e., no agent wants to swap her bundle) and Pareto optimality (where an allocation is Pareto-optimal w.r.t. the agents' preferences if no other allocation can make some agent better off without making some other agent worse off). For example, they show that Borda-optimal allocations (w.r.t. the "leximin order") are always possibly Pareto-optimal, whereas they in general fail to be necessarily Pareto-optimal-notions that are closely related to the notions of possible and necessary winner in voting due to Konczak and Lang (2005).

Baumeister et al. (2017) study both axiomatic and computational properties of scoring allocation correspondences and rules. For example, they show that Borda scoring (with egalitarian social welfare and any tie-breaking relation) satisfies monotonicity, yet does not satisfy what they call "global monotonicity," "possible object monotonicity," and "separability." And that, given the agents' preferences, the problem of whether there is an allocation whose egalitarian social welfare exceeds a given value is NP-complete for Borda. Nguyen, Baumeister, and Rothe (2018) characterize strategy-proofness, as defined by Kelly (1977), for scoring allocation correspondences with utilitarian social welfare. For Borda, their result implies that strategy-proofness holds if and only if there are no more than two goods.

\subsection{Forming Coalitions in FEN-Hedonic Games}

Hedonic games, as part of cooperative game theory, model how players, each having preferences about the coalitions they can join, form coalitions. For more background, we refer to the book chapters by Aziz and Savani (2016) and Elkind and Rothe (2015). One problem is how to represent hedonic games, given that each player can join exponentially many (in the number of players) coalitions. Lang et al. (2015) list a number of approaches from the literature for how to deal with this problem, e.g., the friend- and enemyoriented encodings due to Dimitrov et al. (2006). Lang et al. (2015) extend their approach by also allowing neutral players and define FEN-hedonic games: Each player parti-

\footnotetext{
${ }^{8}$ Unlike for Borda in voting, they here use the scoring vector $(m, m-1, \ldots, 1)$ to ensure that each good has some positive value. In voting, such a shift of scores would not matter (Hemaspaandra and Hemaspaandra 2007). For the allocation problem, however, Baumeister et al. (2017) show that such a shift actually does matter.
} 
tions the set of other players into friends, enemies, and neutral players and ranks her friends and enemies. They assume preferences to be monotonic w.r.t. adding friends and antimonotonic w.r.t. adding enemies and use "bipolar responsive extensions" to lift the players' rankings of players to their partial preferences over coalitions. Rothe, Schadrack, and Schend (2018) (see also Section 5.2 in the conference version by Lang et al. (2015)) then employ cardinal comparability functions based on scoring vectors so as to extend partial to complete preference orders consistent with these bipolar responsive orders. Focusing on Borda-induced FEN-hedonic games, they study the complexity of the existence and the verification problem for common solution concepts; e.g., verifying "Nash stability" is in $\mathrm{P}$ and testing if a Nash-stable coalition structure exists is NP-complete, while verifying "core stability" is coNP-complete and testing if a core-stable coalition structure exists is $\mathrm{NP}^{\mathrm{NP}}$-complete.

\section{Conclusions}

We have summarized recent results on how Borda has been used in collective decision making, ranging from voting (in particular, manipulation, control, and bribery in elections) to other fields (allocating indivisible goods to agents and hedonic games). This wide range of applicability is quite astonishing, considering how simple and elegant Borda's rule is.

We have also surveyed the most central models in computational social choice, a true success story within AI, and have mentioned some of the most important results for other voting rules alongside Borda. While most questions for Borda have been settled by now, there are still some open issues, e.g., the question mark in Table 1 for DCPC-TP.

Establishing hardness in typical settings rather than merely worst-case hardness results is still a great challenge in manipulation, control, and bribery. More generally, we propose to keep looking for new applications of this vintage voting rule in other fields and domains.

\section{Acknowledgements}

This work was supported by DFG grant RO 1202/14-2.

\section{References}

Aziz, H., and Savani, R. 2016. Hedonic games. In Brandt, F.; Conitzer, V.; Endriss, U.; Lang, J.; and Procaccia, A., eds., Handbook of Computational Social Choice. Cambridge University Press. chapter 15, 356-376.

Bachrach, Y.; Lev, O.; Lewenberg, Y.; and Zick, Y. 2016. Misrepresentation in district voting. In Proc. IJCAI'16, 81-87. AAAI Press/IJCAI.

Baldwin, J. 1926. The technique of the Nanson preferential majority system of election. Transactions and Proceedings of the Royal Society of Victoria 39:42-52.

Bartholdi III, J., and Orlin, J. 1991. Single transferable vote resists strategic voting. Social Choice and Welfare 8(4):341-354.

Bartholdi III, J.; Tovey, C.; and Trick, M. 1989. The computational difficulty of manipulating an election. Social Choice and Welfare 6(3):227-241.

Bartholdi III, J.; Tovey, C.; and Trick, M. 1992. How hard is it to control an election? Math. and Comp. Modelling 16(8/9):27-40.
Baumeister, D., and Rothe, J. 2015. Preference aggregation by voting. In Rothe, J., ed., Economics and Computation. Springer. 197-325.

Baumeister, D.; Bouveret, S.; Lang, J.; Nguyen, N.; Nguyen, T.; Rothe, J.; and Saffidine, A. 2017. Positional scoring-based allocation of indivisible goods. Journal of Autonomous Agents and Multi-Agent Systems 31(3):628-655.

Betzler, N.; Niedermeier, R.; and Woeginger, G. 2011. Unweighted coalitional manipulation under the Borda rule is NP-hard. In Proc. IJCAI'11, 55-60. AAAI Press/IJCAI.

Borda, J. 1781. Mémoire sur les élections au scrutin. Histoire de L'Académie Royale des Sciences, Paris.

Bouveret, S.; Chevaleyre, Y.; and Maudet, N. 2016. Fair allocation of indivisible goods. In Brandt, F.; Conitzer, V.; Endriss, U.; Lang, J.; and Procaccia, A., eds., Handbook of Computational Social Choice. Cambridge University Press. 284-310.

Bouveret, S.; Endriss, U.; and Lang, J. 2010. Fair division under ordinal preferences: Computing envy-free allocations of indivisible goods. In Proc. ECAI'10, 387-392. IOS Press.

Brams, S., and King, D. 2005. Efficient fair division: Help the worst off or avoid envy? Rationality and Society 17(4):387-421.

Brams, S.; Edelman, P.; and Fishburn, P. 2003. Fair division of indivisible items. Theory and Decision 55(2):147-180.

Brelsford, E.; Faliszewski, P.; Hemaspaandra, E.; Schnoor, H.; and Schnoor, I. 2008. Approximability of manipulating elections. In Proc. AAAI'08, 44-49. AAAI Press.

Chen, J.; Faliszewski, P.; Niedermeier, R.; and Talmon, N. 2015. Elections with few voters: Candidate control can be easy. In Proc. AAAI'15, 2045-2051. AAAI Press.

Conitzer, V., and Walsh, T. 2016. Barriers to manipulation in voting. In Brandt, F.; Conitzer, V.; Endriss, U.; Lang, J.; and Procaccia, A., eds., Handbook of Computational Social Choice. Cambridge University Press. 127-145.

Conitzer, V.; Sandholm, T.; and Lang, J. 2007. When are elections with few candidates hard to manipulate? Journal of the ACM 54(3):Article 14.

Davies, J.; Katsirelos, G.; Narodytska, N.; and Walsh, T. 2011. Complexity of and algorithms for Borda manipulation. In Proc. AAAI'11, 657-662. AAAI Press.

Davies, J.; Katsirelos, G.; Narodytska, N.; Walsh, T.; and Xia, L. 2014. Complexity of and algorithms for the manipulation of Borda, Nanson's and Baldwin's voting rules. Artificial Intelligence 217:20-42.

Dimitrov, D.; Borm, P.; Hendrickx, R.; and Sung, S. 2006. Simple priorities and core stability in hedonic games. Social Choice and Welfare 26(2):421-433.

Dwork, C.; Kumar, R.; Naor, M.; and Sivakumar, D. 2001. Rank aggregation methods for the web. In Proc. WWW'01, 613-622. ACM Press.

Elkind, E., and Faliszewski, P. 2010. Approximation algorithms for campaign management. In Proc. WINE'10, 473-482. SpringerVerlag LNCS \#6484.

Elkind, E., and Rothe, J. 2015. Cooperative game theory. In Rothe, J., ed., Economics and Computation. Springer. 135-193.

Elkind, E.; Faliszewski, P.; and Slinko, A. 2009. Swap bribery. In Proc. SAGT'09, 299-310. Springer-Verlag LNCS \#5814.

Elkind, E.; Faliszewski, P.; and Slinko, A. 2011. Cloning in elections: Finding the possible winners. Journal of Artificial Intelligence Research 42:529-573. 
Ephrati, E., and Rosenschein, J. 1997. A heuristic technique for multi-agent planning. Annals of Mathematics and Artificial Intelligence 20(1-4):13-67.

Erdélyi, G.; Fellows, M.; Rothe, J.; and Schend, L. 2015. Control complexity in Bucklin and fallback voting: A theoretical analysis. Journal of Computer and System Sciences 81(4):632-660.

Erdélyi, G.; Hemaspaandra, E.; and Hemaspaandra, L. 2015. More natural models of electoral control by partition. In Proc. ADT'15, 396-413. Springer-Verlag LNAI \#9346.

Faliszewski, P., and Rothe, J. 2016. Control and bribery in voting. In Brandt, F.; Conitzer, V.; Endriss, U.; Lang, J.; and Procaccia, A., eds., Handbook of Computational Social Choice. Cambridge University Press. 146-168.

Faliszewski, P.; Hemaspaandra, E.; Hemaspaandra, L.; and Rothe, J. 2009. Llull and Copeland voting computationally resist bribery and constructive control. Journal of Artificial Intelligence Research 35:275-341.

Faliszewski, P.; Reisch, Y.; Rothe, J.; and Schend, L. 2015. Complexity of manipulation, bribery, and campaign management in Bucklin and fallback voting. Journal of Autonomous Agents and Multi-Agent Systems 29(6):1091-1124.

Faliszewski, P.; Hemaspaandra, E.; and Hemaspaandra, L. 2009. How hard is bribery in elections? Journal of Artificial Intelligence Research 35:485-532.

Faliszewski, P.; Hemaspaandra, E.; and Schnoor, H. 2008. Copeland voting: Ties matter. In Proc. AAMAS'08, 983-990. IFAAMAS

Ghosh, S.; Mundhe, M.; Hernandez, K.; and Sen, S. 1999. Voting for movies: The anatomy of recommender systems. In Proc. 3rd Annual Conference on Autonomous Agents, 434-435. ACM Press.

Gibbard, A. 1973. Manipulation of voting schemes. Econometrica 41(4):587-601.

Haynes, T.; Sen, S.; Arora, N.; and Nadella, R. 1997. An automated meeting scheduling system that utilizes user preferences. In Proc. AGENTS'97, 308-315. ACM Press.

Hemaspaandra, E., and Hemaspaandra, L. 2007. Dichotomy for voting systems. Journal of Computer and System Sciences 73(1):73-83.

Hemaspaandra, E., and Schnoor, H. 2016. Dichotomy for pure scoring rules under manipulative electoral actions. In Proc. ECAI'16, 1071-1079. IOS Press.

Hemaspaandra, E.; Hemaspaandra, L.; and Menton, C. 2013. Search versus decision for election manipulation problems. In Proc. STACS'13, volume 20 of LIPIcs, 377-388. Schloss Dagstuhl - Leibniz-Zentrum für Informatik.

Hemaspaandra, E.; Hemaspaandra, L.; and Rothe, J. 2007. Anyone but him: The complexity of precluding an alternative. Artificial Intelligence 171(5-6):255-285.

Hemaspaandra, E.; Hemaspaandra, L.; and Rothe, J. 2017. The complexity of controlling candidate-sequential elections. Theoretical Computer Science 678:14-21.

Kelly, J. 1977. Strategy-proofness and social choice functions without single-valuedness. Econometrica 45(2):439-446.

Konczak, K., and Lang, J. 2005. Voting procedures with incomplete preferences. In Proc. Multidisciplinary IJCAI-05 Workshop on Advances in Preference Handling, 124-129.

Lang, J., and Rothe, J. 2015. Fair division of indivisible goods. In Rothe, J., ed., Economics and Computation. Springer. 493-550.
Lang, J.; Rey, A.; Rothe, J.; Schadrack, H.; and Schend, L. 2015. Representing and solving hedonic games with ordinal preferences and thresholds. In Proc. AAMAS'15, 1229-1237.

Loreggia, A.; Narodytska, N.; Rossi, F.; Venable, B.; and Walsh, T. 2015. Controlling elections by replacing candidates or votes (extended abstract). In Proc. AAMAS'15, 1737-1738. IFAAMAS. Maushagen, C.; Neveling, M.; Rothe, J.; and Selker, A. 2018. Complexity of shift bribery in iterative elections. In Proc. AAMAS'18, 1567-1575. IFAAMAS.

Menton, C. 2013. Normalized range voting broadly resists control. Theory of Computing Systems 53(4):507-531.

Nanson, E. 1882. Methods of election. Transactions and Proceedings of the Royal Society of Victoria 19:197-240.

Neveling, M., and Rothe, J. 2017a. Closing the gap of control complexity in Borda elections: Solving ten open cases. In Proc. ICTCS'17, volume 1949, 138-149. CEUR-WS.org.

Neveling, M., and Rothe, J. 2017b. Solving seven open problems of offline and online control in Borda elections. In Proc. AAAI'17, 3029-3035. AAAI Press.

Nguyen, N.; Baumeister, D.; and Rothe, J. 2018. Strategyproofness of scoring allocation correspondences for indivisible goods. Social Choice and Welfare 50(1):101-122.

Oflazer, K., and Tür, G. 1997. Morphological disambiguation by voting constraints. In Proc. EACL'97, 222-229. ACL Press.

Pennock, D.; Horvitz, E.; and Giles, C. 2000. Social choice theory and recommender systems: Analysis of the axiomatic foundations of collaborative filtering. In Proc. AAAI'00, 729-734. AAAI Press. Puppe, C., and Tasnádi, A. 2009. Optimal redistricting under geographical constraints: Why "pack and crack" does not work. Economics Letters 105(1):93-96.

Risse, M. 2005. Why the count de Borda cannot beat the Marquis de Condorcet. Social Choice and Welfare 25:95-113.

Rothe, J., and Schend, L. 2013. Challenges to complexity shields that are supposed to protect elections against manipulation and control: A survey. Annals of Mathematics and Artificial Intelligence 68(1-3):161-193.

Rothe, J.; Schadrack, H.; and Schend, L. 2018. Borda-induced hedonic games with friends, enemies, and neutral players. Mathematical Social Sciences 96:21-36.

Russel, N. 2007. Complexity of control of Borda count elections. Master's thesis, Rochester Institute of Technology.

Saari, D. 2003. Capturing the 'Will of the People'. Ethics 113:333334.

Saari, D. 2006. Which is better: the Condorcet or Borda winner? Social Choice and Welfare 27(1):107-129.

Satterthwaite, M. 1975. Strategy-proofness and Arrow's conditions: Existence and correspondence theorems for voting procedures and social welfare functions. Journal of Economic Theory 10(2):187-217.

Sigletos, G.; Paliouras, G.; Spyropoulos, C.; and Hatzopoulos, M. 2005. Combining information extraction systems using voting and stacked generalization. Journal of Machine Learning Research 6:1751-1782.

Zuckerman, M.; Procaccia, A.; and Rosenschein, J. 2009. Algorithms for the coalitional manipulation problem. Artificial Intelligence 173(2):392-412.

Zwicker, W. 2016. Introduction to the theory of voting. In Brandt, F.; Conitzer, V.; Endriss, U.; Lang, J.; and Procaccia, A., eds. Handbook of Computational Social Choice. Cambridge University Press. chapter 2, 23-56. 